\title{
A LUMINOUS X-RAY FLARE FROM THE NUCLEUS OF THE DORMANT BULGELESS SPIRAL GALAXY NGC 247
}

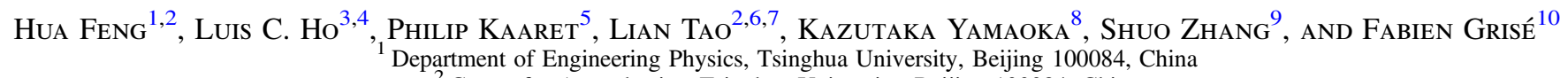 \\ ${ }^{2}$ Center for Astrophysics, Tsinghua University, Beijing 100084, China \\ ${ }^{3}$ Kavli Institute for Astronomy and Astrophysics, Peking University, Beijing 100871, China \\ ${ }^{4}$ Department of Astronomy, School of Physics, Peking University, Beijing 100871, China \\ ${ }^{5}$ Department of Physics and Astronomy, University of Iowa, Iowa City, IA 52242, USA \\ ${ }^{6}$ Cahill Center for Astronomy and Astrophysics, California Institute of Technology, Pasadena, CA 91125, USA \\ ${ }^{7}$ Department of Physics, Tsinghua University, Beijing 100084, China \\ ${ }^{8}$ Department of Physics and Mathematics, Aoyama Gakuin University, 5-10-1 Fuchinobe, Chuo-ku, Sagamihara 252-5258, Japan \\ ${ }_{9}^{9}$ Columbia Astrophysics Laboratory, Columbia University, New York, NY 10027, USA \\ ${ }^{10}$ Observatoire Astronomique de Strasbourg, Université de Strasbourg, CNRS, UMR 7550, 11 rue de l'Université, F-67000 Strasbourg, France \\ Received 2015 March 15; accepted 2015 June 1; published 2015 July 10
}

\begin{abstract}
NGC 247 is a nearby late-type bulgeless spiral galaxy that contains an inactive nucleus. We report a serendipitous discovery of an X-ray flare from the galaxy center with a luminosity of up to $2 \times 10^{39} \mathrm{erg} \mathrm{s}^{-1}$ in the $0.3-10 \mathrm{keV}$ band with XMM-Newton. A Chandra observation confirms that the new X-ray source is spatially coincident with the galaxy nucleus. The XMM-Newton data revealed a hard power-law spectrum with a spectral break near 3-4 keV, no pulsations on timescales longer than $150 \mathrm{~ms}$, and a flat power spectrum consistent with Poisson noise from $1 \mathrm{mHz}$ to nearly $10 \mathrm{~Hz}$. Follow-up observations with Swift detected a second flux peak followed by a luminosity drop by a factor of almost 20. The spectral and temporal behaviors of the nuclear source are consistent with the scenario that the flare was due to an outburst of a low-mass X-ray binary that contains a stellar-mass black hole emitting near its Eddington limit at the peak. However, it cannot be ruled out that the sudden brightening in the nucleus was due to accretion onto a possible low-mass nuclear black hole, fed by a tidally disrupted star or a gas cloud; the Monitor of All-sky X-ray Image observations limit the peak luminosity of the flare to less than $\sim 10^{43} \mathrm{erg} \mathrm{s}^{-1}$, suggesting that it is either a low-mass black hole or an inefficient tidal disruption event.
\end{abstract}

Key words: galaxies: nuclei - X-rays: galaxies - black hole physics

\section{INTRODUCTION}

It is widely accepted that most massive galaxies harbor supermassive black holes (for reviews, see Ho 2008; Kormendy \& Ho 2013). However, it is still unknown what fraction of small, bulgeless galaxies contains nuclear black holes (for a review see Greene 2012). If they do, they are most likely to harbor low-mass black holes with masses $\lesssim 10^{6} M_{\odot}$ or even less (e.g., Filippenko \& Ho 2003; Barth et al. 2004; Peterson et al. 2005; Seth et al. 2010; Reines et al. 2011). Current searches for nuclear black holes in small galaxies were mainly done in optical (Greene \& Ho 2004, 2007b; Barth et al. 2008; Izotov \& Thuan 2008; Dong et al. 2012; Izotov et al. 2012). These means are of low efficiency because they only allow us to find the most active nuclei in nearby galaxies. X-ray observations help to detect X-ray cores with much lower levels of activity, but the physical nature of the X-ray cores is uncertain (Desroches \& Ho 2009; Lemons et al. 2015). Meanwhile, the active fraction goes down toward the low-mass end for nuclear black holes (Greene \& Ho 2007a).

For the majority of inactive low-mass galaxies, the tidal disruption event (TDE; Rees 1988) could be a powerful probe for their discovery and in-depth study. TDEs may allow us to census black holes in dormant galaxies and even estimate the black hole mass and spin with detailed X-ray spectroscopy and timing. A couple dozen of such events, or suspected events, have been reported to date (for a review, see Komossa 2012). The TDE rate was estimated to be of the order of $10^{-5}-10^{-4}$ per year per galaxy, based on possible detections in X-ray surveys with ROSAT (Donley et al. 2002), XMM-Newton (Esquej et al. 2007), or Chandra (Luo et al. 2008). Thus it would have been very lucky to catch a TDE in or around our local group. The chance may be enhanced in low-mass galaxies because, theoretically, the TDE rate is inversely proportional to the black hole mass, and becomes the highest in nucleated dwarf galaxies if they contain black holes (Wang \& Merritt 2004). Indeed, two candidate TDEs have been detected in dwarf galaxies, WINGS $\mathrm{J} 1348$ in the galaxy cluster Abell $1795(z=0.06$; Maksym et al. 2013, 2014b; Donato et al. 2014) and RBS 1032 associated with SDSS J114726.69+494257.8 $\quad(z=0.026$; Maksym et al. 2014a), both located more than $100 \mathrm{Mpc}$ away.

Here, we report a serendipitous detection of a strong-X-ray flare from the nucleus of NGC 247, which is a nearby dormant galaxy. If it is indeed a nuclear black hole event, then this source offers us a unique case besides our Galactic center for studying accretion onto quiescent black holes due to its proximity and, if it is a TDE, will allow us to investigate the TDE behavior in its late phase. On the other hand, the flare could be due to an outburst from an unresolved stellar-mass black hole in the nuclear star cluster. Such objects were excluded in the study of black hole binaries and/or ultraluminous X-ray sources (ULXs) in external galaxies, almost without exception. In this regard, some of these objects, such as the X-ray cores of M33 (La Parola et al. 2003) and NGC 253 (Brunthaler et al. 2009), could be misidentified as weak X-ray active galactic nuclei (AGNs) especially with the lack of multiwavelength information, though their contamination to AGN surveys is estimated to be low (Desroches \& Ho 2009; Gallo et al. 2010). 
NGC 247 is a nearby late-type spiral galaxy classified as $\mathrm{SAB}(\mathrm{s}) \mathrm{d}$ (de Vaucouleurs et al. 1991) in the Sculptor group, which is just outside of our local group. We adopt an IR-based Cepheid distance of 3.4 Mpc to the galaxy (Gieren et al. 2009). The total $\mathrm{H}$ I mass was measured to be $\sim 10^{9} M_{\odot}$ (Carignan \& Puche 1990; Ott et al. 2012). The galaxy has a high inclination angle, $74^{\circ}$, and an isophotal diameter $D_{25}=19.7 \mathrm{kpc}$ assuming the distance mentioned above (Carignan 1985). It has a total apparent $B$ magnitude of about 9.7 (Carignan 1985; Pierce \& Tully 1992). Assuming a Galactic foreground extinction $A_{B}=0.078$ (Schlegel et al. 1998), an internal extinction $A_{B}=0.67$ (Carignan 1985), and a distance modulus of 27.64 (Gieren et al. 2009), the total absolute blue magnitude is $M_{B}^{0}=-18.7$.

NGC 247 contains a nuclear star cluster similar to other latetype spiral galaxies (Davidge \& Courteau 2002). The dynamical center of the galaxy determined via $21 \mathrm{~cm}$ observations was found to be consistent with its optical center (Carignan \& Puche 1990). UV spectroscopy of the nuclear cluster (Maoz et al. 1995) suggests that it is an $\mathrm{H}$ II region with no signature of an AGN. Past X-ray observations also indicate that it is a dormant galaxy.

The star formation rate of the galaxy is estimated to be 0.03 $M_{\odot} \mathrm{yr}^{-1}$ using the $\mathrm{H} \alpha$ emission line (Ferguson et al. 1996). The low star formation rate, and the lack of tidal features in NGC 247 and Histreams between NGC 247 and its neighbor NGC 253 (Davidge 2010), suggest that there is no ongoing or past mergers that could potentially trigger the activity of an AGN.

\section{OBSERVATIONS}

\subsection{Previous Non-detections}

Before 2014, X-ray observations of NGC 247 did not detect any X-ray emission from the nuclear region. A Chandra observation was made on 2011 February 01 (ObsID 12437) with an effective exposure of $5.0 \mathrm{ks}$. We created a new level 2 event file using the repro script in CIAO. We calculated a radius of 1 1".573 that enclosed $90 \%$ of the point-spread function in the nuclear position using the psfsize_srcs command and adopted it as the source aperture. A concentric annular region was used to estimate the background. There was actually no photon detected in the source aperture in $0.3-8 \mathrm{keV}$. The $90 \%$ upper limit of the source flux, based on background fluctuation in the source aperture assuming Poisson distribution, was estimated to be $5.10 \times 10^{-4}$ counts $\mathrm{s}^{-1}$ in $0.3-8 \mathrm{keV}$ using the Bayesian approach with the aprates command. With the local spectral response files, assuming a broken power-law spectrum that was obtained during the flare (see the next paragraph and Table 1), such a count rate can be translated to a $90 \%$ flux upper limit of $1.1 \times 10^{-14} \mathrm{erg} \mathrm{cm}^{-2} \mathrm{~s}^{-1}$ or an absorption-corrected luminosity of $1.8 \times 10^{37} \mathrm{erg} \mathrm{s}^{-1}$ in $0.3-10 \mathrm{keV}$.

The galaxy was observed with XMM-Newton on 2009 December 07 (ObsID 0601010101, see Figure 1). We created new event files with updated calibration files and selected events from intervals where the background flux was within $\pm 3 \sigma$ of the mean quiescent level. The PN data were not used because the nuclear position was located on bad CCD columns. The MOS1 and MOS2 data, with an effective exposure of nearly $30 \mathrm{ks}$, were used to estimate the detection limit with a source and a larger, nearby background region. A source aperture size of $20^{\prime \prime}$ in radius was found to be the optimal choice. Because there are sufficient counts in the aperture, it allows us to use the normal distribution to approximate the Poisson distribution. With spectral responses generated in the nuclear position and assuming the same broken power-law model (see Table 1), we estimate a $90 \%$ upper limit of the observed flux as $4.9 \times 10^{-15} \mathrm{erg} \mathrm{cm}^{-2} \mathrm{~s}^{-1}$ and an absorptioncorrected luminosity of $8.0 \times 10^{36} \mathrm{erg} \mathrm{s}^{-1}$ in $0.3-10 \mathrm{keV}$. Another XMM-Newton observation made on 2001 July 8 does not enable us to place a stringent upper limit due to heavy background contamination.

\subsection{First Detection with XMM-Newton}

The first detection of a bright X-ray source in the nuclear region of NGC 247 was made on 2014 July 1 with XMMNewton (ObsID 0728190101), see Figure 1. Following the same process mentioned above, we created new event files and filtered for background flares, resulting in an effective exposure of $24.3 \mathrm{ks}$. The X-ray energy spectra were extracted from a circular region with a radius of 32 arcsec, and the background spectra were extracted from a nearby circular region on the same CCD chip, using events with FLAG $=0$ and PATTERN $\leqslant 12$ for MOS and $\leqslant 4$ for PN. The spectra were grouped such that each new spectral bin was one-fourth of the local FWHM or had at least 15 net counts, from 0.2 to $10 \mathrm{keV}$ for PN and from 0.3 to $10 \mathrm{keV}$ for MOS. The PN and MOS spectra were loaded into XSPEC v12 jointly. A multiplicative constant for MOS flux relative to the PN flux was introduced as a free parameter to account for possible systematics in their effective area calibration, and was found to be close to 1.05 . The interstellar absorption consists of a Galactic foreground component fixed at $2.06 \times 10^{20} \mathrm{~cm}^{-2}$ (Kalberla et al. 2005) and a free, extragalactic component, both using the TBabs model (Wilms et al. 2000).

We first tried a simple power-law model to fit the spectrum. The fitting is inadequate (a null hypothesis probability of 0.03 while we define $>0.05$ as being acceptable) and a trend of spectral break can be seen in the residual above a few $\mathrm{keV}$. We thus tried a broken power-law model to account for the possible break, which significantly improved the fits and the presence of the break has a significance of $1.3 \times 10^{-12}$ with partial F-test. A comparison of the power-law and broken power-law fits is displayed in Figure 2. An exponentially cut-off power-law model produces a similar goodness of fit. We then tried physical models that allowed for a high-energy cutoff, including the multicolor disk (MCD) model as an approximation to the standard accretion disk model (Shakura \& Sunyaev 1973) and the $p$-free model, with the radial dependence of temperature $\left(T(R) \propto R^{-p}\right)$ being a free parameter, as an approximation to the slim-disk model (Mineshige et al. 1994). We also tested a double-component model, MCD plus powerlaw, which is the standard to fit the X-ray energy spectra of accreting black hole binaries. The spectral parameters along with observed fluxes and absorption-corrected luminosities (computed with the cflux model) are listed in Table 1. The power-law and MCD models cannot provide an adequate fit (null hypothesis probability $<0.05$ ), while the other models can.

The PN data have a continuous good time interval of $26.1 \mathrm{ks}$ (no background flares or timing gaps), allowing us to search for timing signals. Coherent signals were searched in the full band $(0.2-10 \mathrm{keV})$ based on the CCD frame time (about $73 \mathrm{~ms}$ ) with a 35514-point Fourier transform. The highest 
Table 1

X-ray Spectral Fitting to the XMM-Newton Spectrum

\begin{tabular}{|c|c|c|c|c|c|c|c|c|c|c|}
\hline Model & $N_{\mathrm{H}, \mathrm{ext}}{ }^{\mathrm{a}}\left(10^{21} \mathrm{~cm}^{-2}\right)$ & $\Gamma$ & $N_{\mathrm{PL}}{ }^{\mathrm{b}}$ & $E_{\mathrm{b}}{ }^{\mathrm{c}}(\mathrm{keV})$ & $\Gamma_{2} / p^{\mathrm{d}}$ & $T_{\text {in }}(\mathrm{keV})$ & $R_{\text {in }}{ }^{\mathrm{e}}(\mathrm{km})$ & $f_{0.3-10 \mathrm{keV}}\left(10^{12} \mathrm{erg} \mathrm{cm}^{2} \mathrm{~s}^{-1}\right)$ & $L_{0.3-10 \mathrm{keV}}\left(10^{39} \mathrm{erg} \mathrm{cm}^{-2} \mathrm{~s}^{-1}\right)$ & $\chi^{2} / \mathrm{dof}$ \\
\hline Power-law & $1.50 \pm 0.12$ & $1.74 \pm 0.04$ & $2.39 \pm 0.11$ & $\cdots$ & $\cdots$ & $\cdots$ & $\cdots$ & $1.29 \pm 0.03$ & $2.22 \pm 0.05$ & $549.04 / 490$ \\
\hline Broken PL & $1.09 \pm 0.15$ & $1.50 \pm 0.08$ & $2.07 \pm 0.12$ & $3.5 \pm 0.4$ & $2.3 \pm 0.2$ & $\cdots$ & $\cdots$ & $1.23_{-0.02}^{+0.05}$ & $1.98 \pm 0.07$ & $490.79 / 488$ \\
\hline Cutoff PL & $0.78 \pm 0.18$ & $1.07 \pm 0.15$ & $2.35 \pm 0.10$ & $4.7_{-0.9}^{+1.4}$ & $\ldots$ & $\cdots$ & $\ldots$ & $1.22_{-0.02}^{+0.03}$ & $1.87 \pm 0.08$ & $494.63 / 489$ \\
\hline MCD & $0.18 \pm 0.06$ & $\cdots$ & $\cdots$ & $\cdots$ & $\cdots$ & $1.55 \pm 0.05$ & $34 \pm 2$ & $1.15_{-0.03}^{+0.04}$ & $1.63 \pm 0.04$ & $573.00 / 490$ \\
\hline$p$-free & $0.95 \pm 0.17$ & $\cdots$ & $\cdots$ & $\cdots$ & $0.59 \pm 0.02$ & $2.3 \pm 0.3$ & $11 \pm 3$ & $1.21_{-0.02}^{+0.04}$ & $1.91 \pm 0.08$ & $491.89 / 489$ \\
\hline $\mathrm{PL}+\mathrm{MCD}$ & $1.5_{-0.5}^{+0.8}$ & $2.3_{-0.5}^{+0.8}$ & $1.4_{-0.3}^{+0.4}$ & $\ldots$ & $\ldots$ & $1.92_{-0.33}^{+0.16}$ & $19_{-3}^{+4}$ & $1.21_{-0.02}^{+0.03}$ & $2.1_{-0.2}^{+0.6}$ & $490.18 / 488$ \\
\hline
\end{tabular}

$\omega \quad$ Notes. All errors are quoted in the $90 \%$ confidence level.

${ }^{\text {a }}$ Extragalactic absorption column density; an additional absorption component fixed at $N_{\mathrm{H}}=2.06 \times 10^{20} \mathrm{~cm}^{-2}$ to account for Galactic absorption is not shown here.

${ }^{\mathrm{b}}$ Normalization in units of $10^{-4}$ photons $\mathrm{keV}^{-1} \mathrm{~cm}^{-2} \mathrm{~s}^{-1}$ at $1 \mathrm{keV}$.

${ }^{c}$ Break energy for the broken power-law model (bknpower in XSPEC) or the e-folding energy for the cutoff power-law model (cutoffpl in XSPEC).

${ }^{\mathrm{d}} \Gamma_{2}$ is the power-law photon index above the break energy for the broken power-law model; $p$ is the radial dependence of the disk temperature in the $p$-free model.

e Assuming a face-on disk. 


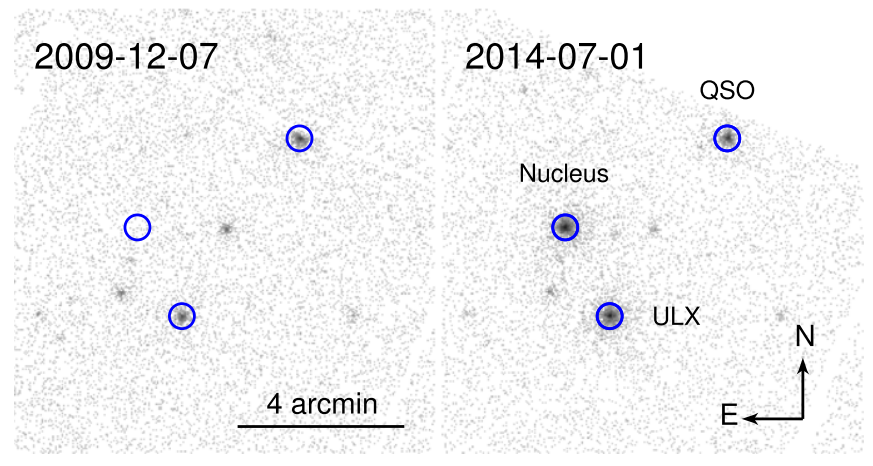

Figure 1. XMM-Newton MOS1 images of the galaxy NGC 247 in 2009 vs. 2014. The galaxy used to show a quiescent nucleus, but a new, bright X-ray source was detected in 2014 and confirmed to be coincident with the galaxy nucleus with a follow-up Chandra observation. The ULX and QSO were used to align Chandra and HST images.

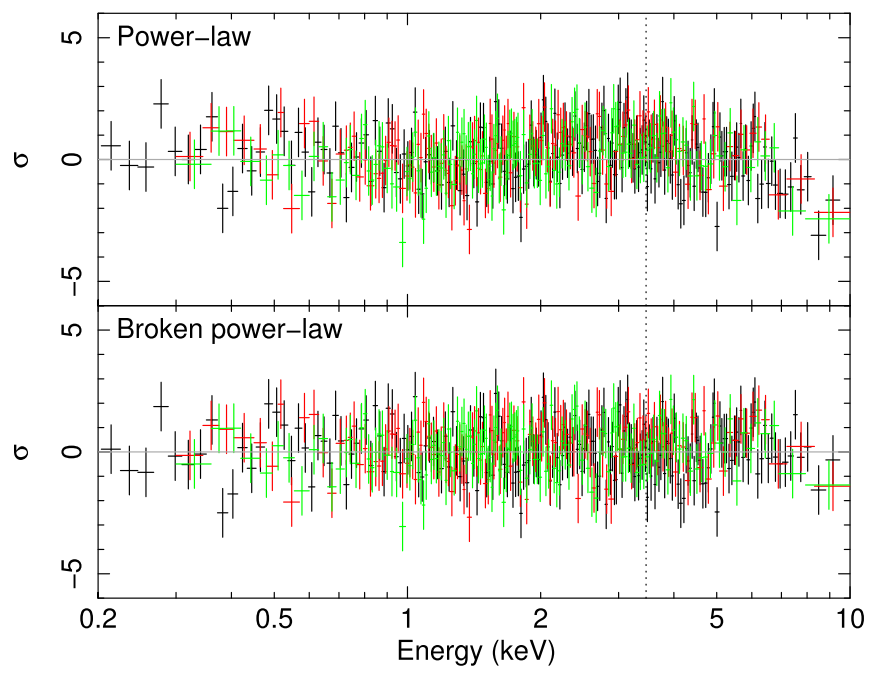

Figure 2. Comparison of the X-ray spectral fitting with the power-law model $\left(\chi^{2}=549.0\right.$ with 490 degrees of freedom $)$ and the broken power-law model $\left(\chi^{2}=490.8\right.$ with 488 degrees of freedom). The dotted line indicates the energy of the spectral break. The residuals after fitting with the power-law model show a curved feature in the $2-10 \mathrm{keV}$ range.

power amplitude is 31.5 at $0.076680 \mathrm{mHz}$ under Leahy normalization (Leahy et al. 1983), while the $99 \%$ confidence level corresponds to an amplitude of 34.8 assuming a $\chi^{2}$ distribution with 2 degrees of freedom and taking into account the number of trials. With the $Z_{1}^{2}$-test (Buccheri et al. 1983), the detected amplitude allows us to place an upper limit on the pulsed fraction to be $4.3 \%$ assuming a sinusoidal signal. Thus, no pulsations on timescales larger than $\sim 150 \mathrm{~ms}$ are found in the data. Searched in different timescales and energy ranges, we did not detect quasi-periodic oscillations or any form of excessive timing noise above the Poisson level. The power spectra in the frequency range from $10^{-3}-10 \mathrm{~Hz}$ are well consistent with Poisson noise. The only possible temporal variation is seen on a timescale near $13.5 \mathrm{ks}$ (Figure 3). However, limited by the total duration of the lightcurve, it is hard to interpret the possible variation.

\subsection{Fine Position with Chandra}

On 2014 November 12, when the source was still active, we conducted a Chandra observation in order to precisely locate its position, to see whether the bright X-ray source found by

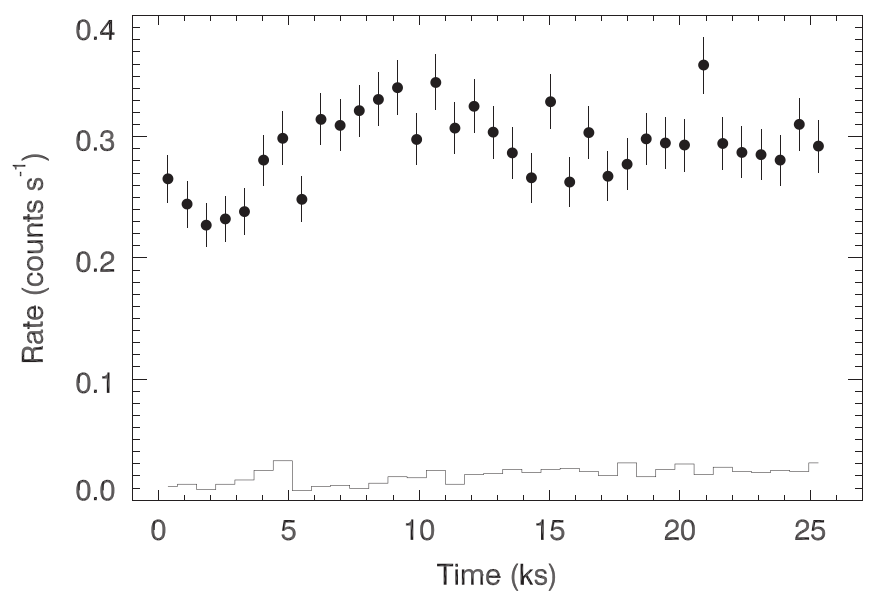

Figure 3. X-ray light curve of the nuclear source. Points are the net count rate and the histogram is the estimated background contribution in the source aperture. The time step is $733.6 \mathrm{~s}$, a binning factor of $10^{4}$ on the CCD frame time. The variation is reflected on the periodogram or the power spectrum as a single peak at the timescale of $13.5 \mathrm{ks}$. A constant fit results in $\chi^{2}=82.2$ with 34 degrees of freedom, corresponding to a chance probability of $7.2 \times 10^{-6}$.

$X M M$-Newton is coincident with the nucleus of the galaxy. The observation was done with the ACIS-S detector and designed to include the new source, an ultraluminous X-ray source (ULX; 2! 4 to its southwest), and a background QSO PHL 6625 ( 4.5 to its northwest; Jin et al. 2011) on the same CCD chip. Both the ULX and the QSO were identified to have a unique optical counterpart (Tao et al. 2012), enabling us to align the Chandra and HST images and improve the relative astrometry. We created exposure-map corrected images and performed source detection with the wavdetectcommand. The source spectrum was extracted using the specextractscript, grouped to be at least 15 counts per energy bin, and fitted with an absorbed power-law model (in the same format as for $X M M-N e w t o n)$ in the energy range of $0.3-8.0 \mathrm{keV}$. The simple power-law model gives an adequate fit $\left(\chi^{2}=26.57\right.$ with 25 degrees of freedom), with an extragalactic absorption column density of $N_{\mathrm{H}}=(3.6 \pm 1.8) \times 10^{21} \mathrm{~cm}^{-2}$, a photon index of $\Gamma=1.9 \pm 0.3$, an observed flux of $(1.14 \pm 0.17) \times 10^{-12}$ $\mathrm{erg} \mathrm{cm}^{-2} \mathrm{~s}^{-1}$, and an intrinsic luminosity of $2.5_{-0.4}^{+0.6} \times 10^{39}$ $\mathrm{erg} \mathrm{s}^{-1}$ in $0.3-10 \mathrm{keV}$. If we adopt the broken power-law model (Table 1) and leave only the normalization as a free parameter, we derive $f_{\mathrm{X}}=(1.10 \pm 0.09) \times 10^{-12} \mathrm{erg} \mathrm{cm}^{-2} \mathrm{~s}^{-1}$ and $L_{\mathrm{X}}=(1.81 \pm 0.14) \times 10^{39} \mathrm{erg} \mathrm{s}^{-1}$ in $0.3-10 \mathrm{keV}, \quad$ with $\chi^{2}=30.35$ and 27 degrees of freedom. All of the errors are at the $90 \%$ confidence level.

An HST image covering the three $\mathrm{X}$-ray sources was created using archival ACS WFC F606W images with the multidrizzle task. Using the geomap task in IRAF, we registered the X-ray positions of the ULX and the QSO to the HST positions of their optical counterparts, with shifts in $\mathrm{X}$ and $\mathrm{Y}$ and a rotation. Then the geoxytran task was used to transform the X-ray position of the nuclear source onto the HST coordinates. The resultant position uncertainty includes the statistical errors of the X-ray positions from wavdetect and the coordinate transformation errors from the geomap fitting, and was calculated to be 0 ". 12 in the $90 \%$ confidence level (assuming a Rayleigh distribution). We note that a very similar result is obtained if the alignment is done only with the 


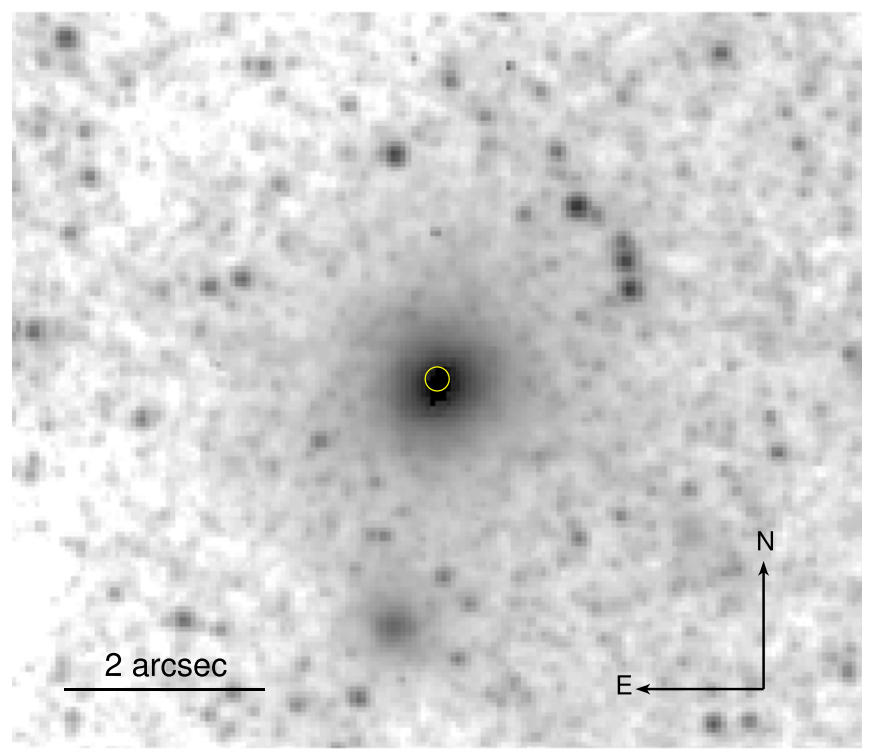

Figure 4. HST ACS WFC F606W image around the NGC 247 nucleus with the corrected X-ray position of the nuclear source, which has an error radius of 0 ". 12. The X-ray source is spatially coincident with the nuclear star cluster.

ULX, which is closer to the nucleus. The corrected position of the nuclear source on the HST image is displayed in Figure 4.

\subsection{Follow-up Monitoring With Swift and the Long-term Lightcurve}

We started a weekly monitoring program for the activity of the nuclear source with the Swift X-ray telescope (XRT) from 2014 October 13 to 2015 May 19. The source was unobservable with Swift for three months toward the end of the monitoring due to Sun avoidance. The effective exposure of each observation varied from 1.3 to $2.5 \mathrm{ks}$. For each observation, we extracted the source spectrum from an aperture of 20 pixel radius, and a background spectrum from a nearby source-free region, using the event file delivered from the pipeline. The detected number of photons in the source aperture ranged from 4 to 52, and the background contribution was less than $40 \%$ in the faintest case. Each spectrum was grouped into two energy bins, one in $0.3-2 \mathrm{keV}$ and the other in $2-10 \mathrm{keV}$. To estimate the source luminosity, we fitted every spectrum in the Cash statistics with the broken power-law model derived from XMM-Newton (Table 1) by fixing all parameters except the normalization. The absorption-corrected luminosity was computed with cflux in $0.3-10 \mathrm{keV}$. If the source was not detected above $1 \sigma$, its $90 \%$ upper limit was calculated using the same Bayesian approach described above.

The X-ray lightcurve (Figure 5) displayed a luminosity change by a factor of 17 . To be consistent, we adopted the Chandra luminosity that was computed assuming the broken power-law model. The irregular variation of the source luminosity and the short baseline do not allow us to fit a power-law decay model to the lightcurve. The Swift lightcurve appears like a fast rise and exponential decay, typical of the outburst behavior of an accreting low-mass X-ray binary. We therefore fitted an exponential decay function, $L=L_{0} \exp \left[-\left(t-t_{0}\right) / \tau\right]$, to the data after the MJD 56960 and derived a decay timescale of $(30 \pm 3)$ days.

Along with the XRT monitoring, the UVOT was in operation with filters UVW1, UVW2, and UVM2 by turns.

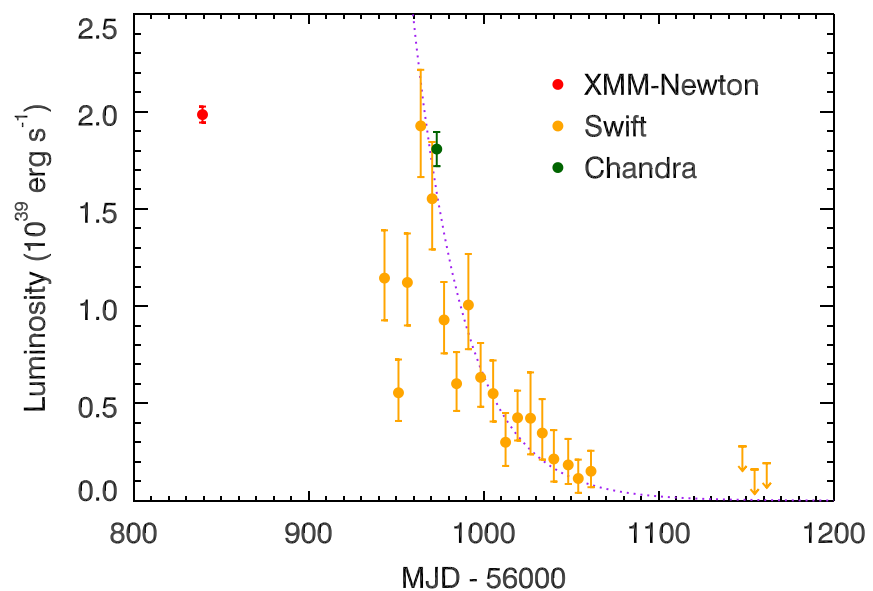

Figure 5. 0.3-10 keV X-ray lightcurve of the NGC 247 nucleus. The dashed line is an exponential decay function, $L=L_{0} \exp \left[-\left(t-t_{0}\right) / \tau\right]$, fitted to the data after MJD 56960.

The source displayed a constant UV flux. The mean flux density corrected for Galactic reddening $(E(B-V)=0.018$; Schlegel et al. 1998) is $1.3 \times 10^{-15} \mathrm{erg} \mathrm{cm}^{-2} \mathrm{~s}^{-1} \AA^{-1}$ in UVW2 (central wavelength at $1928 \AA$ ), $1.2 \times 10^{-15} \mathrm{erg} \mathrm{cm}^{-2} \mathrm{~s}^{-1}$ $\AA^{-1}$ in UVM2 (at $2246 \AA$ ), and $9.7 \times 10^{-16} \mathrm{erg} \mathrm{cm}^{-2} \mathrm{~s}^{-1}$ $\AA^{-1}$ in UVW1 (at $2600 \AA$ ), measured via a source aperture of $3^{\prime \prime}$ and multiple circular background apertures surrounding it. The total luminosity in these UV bands $(\sim 1500-3000 \AA)$ is roughly $3 \times 10^{39} \mathrm{erg} \mathrm{s}^{-1}$. These UV fluxes are consistent with those measured with the Optical Monitor on board XMMNewton and arise from the nuclear star cluster (Maoz et al. 1995), suggesting that no detectable UV emission was found to be associated with the X-ray activity.

\subsection{Search in the Monitor of the All-sky $X$-ray Image (MAXI) Archive}

We searched X-ray emission from the NGC 247 direction with the MAXI on board the international space station (Matsuoka et al. 2009). The search was done with the gas slit camera (GSC) and the solid-state slit camera (SSC) in a variety of energy bands (e.g., $0.7-4,2-4,2-6$, or $2-10 \mathrm{keV}$ ) and timescales (e.g., 1 day, 7 days, or 30 days), in the time window before the first detection with XMM-Newton and the previous non-detection with Chandra (MJD 55594-56839). However, no detection over a significance of $3 \sigma$ can be found with MAXI. The non-detection allows us to place a $3 \sigma$ flux upper limit on the peak flux averaged over one week of $5 \times 10^{-11} \mathrm{erg} \mathrm{cm}^{-2} \mathrm{~s}^{-1}$ in $2-10 \mathrm{keV}$ (with GSC) or $3 \times 10^{-10}$ erg cm${ }^{-2} \mathrm{~s}^{-1}$ in $0.7-2 \mathrm{keV}$ (with SSC). Provided the broken power-law spectrum measured with XMM-Newton, the upper limit in flux can be translated to an upper limit in luminosity in $0.3-10 \mathrm{keV}$ of a few times $10^{41} \mathrm{erg} \mathrm{s}^{-1}$ at a distance of NGC 247 corrected for absorption. If we assume a softer spectrum, e.g., a disk blackbody with an inner temperature of $0.1 \mathrm{keV}$ and an absorption column density of $10^{21} \mathrm{~cm}^{-2}$, the upper limit in luminosity could be as high as $3 \times 10^{43} \mathrm{erg} \mathrm{s}^{-1}$ in the energy band of $0.3-10 \mathrm{keV}$, constrained by the SSC upper limit.

\section{DISCUSSION}

In this paper, we report the detection of a luminous X-ray flare from a compact object in the nuclear star cluster (also the 
dynamical center) of the nearby late-type galaxy NGC 247, which is known to be inactive in both optical and X-ray. There are two possible interpretations of such a sudden brightening in a galaxy nucleus: due to an outburst of a low-mass X-ray binary that resides in the nuclear star cluster, or accretion activity onto a nuclear black hole, such as a TDE.

If not spatially coincident with the galaxy nucleus, this object can be naturally explained as a stellar-mass black hole accreting from a Roche-lobe overflowed low-mass companion star. The luminosity seems to peak around $2 \times 10^{39} \mathrm{erg} \mathrm{s}^{-1}$, which is the Eddington limit of a 10-20 solar mass black hole. A few neutron star binaries have produced an apparent luminosity this high (Skinner et al. 1982; Bachetti et al. 2014). The non-detection of pulsed emission in the XMM-Newton data suggests that it could be a neutron star only if the pulsed fraction is lower than $4 \%$ or the period is shorter than $146 \mathrm{~ms}$. The light curve, especially at times after the peak around MJD 56970, seems to follow a simple exponential decay on a timescale of a month or so, which is often seen in low-mass X-ray binaries as the first phase of flux decay during an outburst (Lasota 2001). The measured decay timescale is roughly consistent with what we have observed for Galactic black hole binaries (see Figures 5-8 in Remillard \& McClintock 2006).

The presence of a spectral break at a few $\mathrm{keV}$ has been ubiquitously found in bright ULXs and argued as a signature of super-Eddington accretion (Stobbart et al. 2006; Gladstone et al. 2009; Motch et al. 2014). Specifically, depending on the MCD plus power-law fitting, this source is typical of a subclass of ULXs, dubbed as the broadened disk class by Sutton et al. (2013), which have relatively low luminosities (near the luminosity threshold for ULXs) and were interpreted as being due to normal stellar-mass black holes $\left(\lesssim 20 M_{\odot}\right)$ accreting near the Eddington level. We argue that the MCD plus powerlaw fit is not physical because the power-law component, which is presumably produced by up-scattering of disk photons in an optically thin corona, dominates over the MCD component at low energies (below $1.5 \mathrm{keV}$ ). The $p$-free model gives a self-consistent interpretation of its nature, being a slimdisk where advection leads to a flattened radial temperature profile $(p=0.6$ versus $p=0.75$ for a standard disk). The derived temperature and luminosity are consistent with emission from a stellar-mass black hole with near-Eddington accretion. The absence of timing noise is also consistent with the spectrum being dominated by emission from a thermal slim-disk.

According to the Chandra survey of ULXs in nearby galaxies (Swartz et al. 2011), we estimated a probability of roughly 0.01 of finding a ULX above $10^{39} \mathrm{erg} \mathrm{s}^{-1}$ within 0 ". 12 at the center of NGC 247. However, such an estimate may be highly uncertain because all objects in their sample are non-nuclear sources by definition. Also, most ULXs were found to have a displacement with star clusters (Kaaret et al. 2004), suggesting a lower probability of finding a ULX associated with a star cluster.

An alternative explanation is that the X-ray flare is due to accretion onto a nuclear black hole of the galaxy, by a tidally disrupted star or other source of gas. In the scenario of a TDE, part of the disrupted stellar materials will be accreted onto the black hole and produce electromagnetic radiation. Normal TDEs (not jetted ones) may have two characteristic features in their early phase, that they may follow a power-law decay in luminosity with an index of $-5 / 3\left(L \propto t^{-5 / 3}\right.$; Rees 1988; Komossa \& Bade 1999; Halpern et al. 2004), and their emission is peaked in the UV and/or soft X-ray band (cf. Komossa 2012) as predicted by the standard accretion disk model. This is in general consistent with observations from the current, limited sample, in which most, if not all, TDEs were recorded in their early phase and the search strategy favored objects bright in UV and soft X-rays. However, the nondetection in the UV band, with a UV luminosity at least one order of magnitude lower than that of the star cluster, i.e., $<$ $\sim 10^{38} \mathrm{erg} \mathrm{s}^{-1}$, stands out as an argument against the early-phase TDE explanation.

The source displayed an overall decreasing trend in flux (Figure 5). However, the short baseline of the monitoring program does not allow for a test of the $t^{-5 / 3}$ power-law decay model, as short-term variability is always associated with an accretion system. Some TDE candidates did show strong shorttimescale variability superposed on a $t^{-5 / 3}$ long-term temporal evolution (e.g., SDSS J1201+30; Saxton et al. 2012). The $\mathrm{X}$-ray energy spectrum is hard, unlike canonical TDE candidates. This could be possible if we had detected it in a late phase. Due to the absence of sensitive observations between 2011 February and 2014 July, we may have caught it one to three years after the initial burst. In this case, the current accretion rate is rather low, the disk is no longer a standard disk, and the emergent spectrum may have become hard. In fact, the TDE candidate IGR J12580+0134 associated with NGC 4845 showed a hard spectrum at its luminosity peak (Nikołajuk \& Walter 2013). Also, the jetted TDE candidates manifested themselves as an extreme subclass with complex spectral and temporal behaviors (Levan et al. 2011; Cenko et al. 2012). These indicate that TDEs may be a diverse population.

The upper limit obtained from the MAXI data indicates that the black hole mass assuming an Eddington limited TDE is less than $\sim 10^{3} M_{\odot}$, if it had emitted a hard spectrum $(\Gamma \lesssim 2)$ from the very beginning. If the initial $\mathrm{X}$-ray spectrum is much softer and peaked at energies below $2 \mathrm{keV}$, then the upper limit in luminosity is much higher, e.g., $\sim 10^{43} \mathrm{erg} \mathrm{s}^{-1}$ given a cool disk emission with $k T_{\text {in }} \approx 0.1 \mathrm{keV}$, and so is the allowed black hole mass (up to $\sim 10^{5} M_{\odot}$ ). Therefore, the Eddington-limited-TDE interpretation may be possible only if the source had transitioned from a luminous soft state to a low hard state, and the central compact object is a low-mass black hole less than a few times $10^{5} M_{\odot}$. Again, TDEs may be significantly sub-Eddington at their luminosity peaks, down to $\sim 10^{-2}$ even assuming the lowest black hole mass estimation ( $\mathrm{Li}$ et al. 2002; Esquej et al. 2008). In that case, the upper limit on the black hole mass estimated with MAXI data could increase by two orders of magnitude.

The two known TDE candidates associated with dwarf galaxies, WINGS J1348 (Maksym et al. 2013; Donato et al. 2014) and RBS 1032 (Maksym et al. 2014a), were both detected at a peak luminosity close to $10^{43} \mathrm{erg} \mathrm{s}^{-1}$ and had undergone a dramatic flux decay by a factor of about two orders of magnitude. WINGS J1348 displayed a luminosity around $10^{41} \mathrm{erg} \mathrm{s}^{-1} 6$ years after its discovery, and so did RBS 1032 almost 20 years after its first detection. They emitted a soft blackbody-like spectrum throughout the active cycle with a temperature near $100 \mathrm{eV}$. The current activity of the object in NGC 247 cannot be longer than 3.5 years, but its spectrum has been hard and the luminosity has gone below $10^{39} \mathrm{erg} \mathrm{s}^{-1}$. 
Thus, this object is at least not in the same class as the two TDEs found in dwarfs.

X-ray observations of the Galactic center molecular clouds indicate that the central supermassive black hole Sgr $A^{\star}$ may also have exhibited giant X-ray flares of similar luminosities in the past a few centuries (for a review see Ponti et al. 2013). Specifically, the X-ray emission from the molecular cloud Sgr B2 requires X-ray flaring activities from $\mathrm{Sgr} \mathrm{A}^{\star}$ which have a power-law spectrum with a photon index of $\Gamma \approx 2$ and an X-ray luminosity of roughly $10^{39} \mathrm{erg} \mathrm{s}^{-1}$ lasting a few years (e.g., Terrier et al. 2010). The spectrum and luminosity are similar to those observed for NGC 247. In addition to the possibility of being triggered by a tidally disrupted star, the accretion materials could come from a stream of gas similar to the case of the G2 cloud near Sgr $A^{\star}$ (Pfuhl et al. 2015), in which case the accretion rate may be insufficient to reach the Eddington limit.

Thus, the temporal and spectral characteristics of this object can be well explained by a stellar-mass black hole accreting close to the Eddington limit at its luminosity peak. However, we emphasize that the possibility for nuclear black hole activity cannot be completely ruled out. The absence of sensitive X-ray observations in the early phase of the flare hampers a conclusive diagnosis of the source nature. Also, the TDE behavior may be too complex to predict especially in its late phase. Plus, the trigger could be other sources like a small gas cloud rather than a disrupted star, whose consequent accretion behavior is even more difficult to predict. A continued X-ray monitoring will be a key to distinguishing the different scenarios. If this object is proven to be a nuclear black hole, it will have great interest in two aspects. First, its proximity will offer us a unique test laboratory for TDE physics at the late phase, if it is a TDE. Also, it suggests that monitoring in X-rays for flaring activity may be an effective tool for us to unveil hidden nuclear black holes in nearby quiescent galaxies.

We thank the anonymous referee for helpful comments that improved the paper, the Chandra director for granting discretionary time and the Swift PI for approving the ToO program, and the XMM-Newton/Chandra/Swift teams for successful executions of the observations. H.F. acknowledges funding support from the National Natural Science Foundation of China under grant No.11222327 and the Tsinghua University Initiative Scientific Research Program. L.C.H. acknowledges support by the Chinese Academy of Science through grant No. XDB09030102 (Emergence of Cosmological Structures) from the Strategic Priority Research Program and by the National Natural Science Foundation of China through grant No.11473002.

Facilities: XMM, CXO, Swift

\section{REFERENCES}

Bachetti, M., Harrison, F. A., Walton, D. J., et al. 2014, Natur, 514, 202 Barth, A. J., Greene, J. E., \& Ho, L. C. 2008, AJ, 136, 1179 Barth, A. J., Ho, L. C., Rutledge, R. E., \& Sargent, W. L. W 2004, ApJ, 607, 90 Brunthaler, A., Castangia, P., Tarchi, A., et al. 2009, A\&A, 497, 103 Buccheri, R., Bennett, K., Bignami, G. F., et al. 1983, A\&A, 128, 245 Carignan, C. 1985, ApJS, 58, 107

Carignan, C., \& Puche, D. 1990, AJ, 100, 641
Cenko, S. B., Krimm, H. A., Horesh, A., et al. 2012, ApJ, 753, 77

Davidge, T. J. 2010, ApJ, 725, 1342

Davidge, T. J., \& Courteau, S. 2002, AJ, 123, 1438

Desroches, L.-B., \& Ho, L. C. 2009, ApJ, 690, 267

de Vaucouleurs, G., de Vaucouleurs, A., Corwin, H. G., Jr., et al. 1991, in

Third Reference Catalogue of Bright Galaxies, Vol. 3 (New York: Springer)

Donato, D., Cenko, S. B., Covino, S., et al. 2014, ApJ, 781, 59

Dong, X.-B., Ho, L. C., Yuan, W., et al. 2012, ApJ, 755, 167

Donley, J. L., Brandt, W. N., Eracleous, M., \& Boller, T. 2002, AJ, 124, 1308

Esquej, P., Saxton, R. D., Freyberg, M. J., et al. 2007, A\&A, 462, L49

Esquej, P., Saxton, R. D., Komossa, S., et al. 2008, A\&A, 489, 543

Ferguson, A. M. N., Wyse, R. F. G., Gallagher, J. S., III, \& Hunter, D. A. 1996, AJ, 111,2265

Filippenko, A. V., \& Ho, L. C 2003, ApJL, 588, L13

Gallo, E., Treu, T., Marshall, P. J., et al. 2010, ApJ, 714, 25

Gieren, W., PietrzyŃski, G., SoszyŃski, I., et al. 2009, ApJ, 700, 1141

Gladstone, J. C., Roberts, T. P., \& Done, C. 2009, MNRAS, 397, 1836

Greene, J. E. 2012, NatCo, 3, 1304

Greene, J. E., \& Ho, L. C. 2004, ApJ, 610, 722

Greene, J. E., \& Ho, L. C. 2007a, ApJ, 667, 131

Greene, J. E., \& Ho, L. C. 2007b, ApJ, 670, 92

Halpern, J. P., Gezari, S., \& Komossa, S. 2004, ApJ, 604, 572

Ho, L. C. 2008, ARA\&A, 46, 475

Izotov, Y. I., \& Thuan, T. X. 2008, ApJ, 687, 133

Izotov, Y. I., Thuan, T. X., \& Privon, G. 2012, MNRAS, 427, 1229

Jin, J., Feng, H., Kaaret, P., \& Zhang, S.-N. 2011, ApJ, 737, 87

Kaaret, P., Alonso-Herrero, A., Gallagher, J. S., et al. 2004, MNRAS, 348, L28

Kalberla, P. M. W., Burton, W. B., Hartmann, D., et al. 2005, A\&A, 440, 775

Komossa, S. 2012, European Physical Journal Web of Conferences, 39, 02001

Komossa, S., \& Bade, N. 1999, A\&A, 343, 775

Kormendy, J., \& Ho, L. C. 2013, ARA\&A, 51, 511

La Parola, V., Damiani, F., Fabbiano, G., \& Peres, G. 2003, ApJ, 583, 758

Lasota, J.-P. 2001, NewAR, 45, 449

Leahy, D. A., Darbro, W., Elsner, R. F., et al. 1983, ApJ, 266, 160

Lemons, S., Reines, A., Plotkin, R., Gallo, E., \& Greene, J. 2015, ApJ, 805, 12 Levan, A. J., Tanvir, N. R., Cenko, S. B., et al. 2011, Sci, 333, 199

Li, L.-X., Narayan, R., \& Menou, K. 2002, ApJ, 576, 753

Luo, B., Brandt, W. N., Steffen, A. T., \& Bauer, F. E. 2008, ApJ, 674, 122

Maksym, W. P., Lin, D., \& Irwin, J. A. 2014a, ApJL, 792, L29

Maksym, W. P., Ulmer, M. P., Eracleous, M. C., Guennou, L., \& Ho, L. C. 2013, MNRAS, 35, 1904

Maksym, W. P., Ulmer, M. P., Roth, K. C., et al. 2014b, MNRAS, 444, 866

Maoz, D., Filippenko, A. V., Ho, L. C., et al. 1995, ApJ, 440, 91

Matsuoka, M., Kawasaki, K., Ueno, S., et al. 2009, PASJ, 61, 999

Mineshige, S., Hirano, A., Kitamoto, S., Yamada, T. T., \& Fukue, J. 1994, ApJ, 426, 308

Motch, C., Pakull, M. W., Soria, R., Grisé, F., \& PietrzyŃski, G. 2014, Natur, 514, 198

Nikołajuk, M., \& Walter, R. 2013, A\&A, 552, A75

Ott, J., Stilp, A. M., Warren, S. R., et al. 2012, AJ, 144, 123

Peterson, B. M., Bentz, M. C., Desroches, L.-B., et al. 2005, ApJ, 632, 799

Pfuhl, O., Gillessen, S., Eisenhauer, F., et al. 2015, ApJ, 798, 111

Pierce, M. J., \& Tully, R. B. 1992, ApJ, 387, 47

Ponti, G., Morris, M. R., Terrier, R., \& Goldwurm, A. 2013, in ASSP Proc. 34, Cosmic Rays in Star-Forming Environments (Berlin: Springer), 331

Rees, M. J. 1988, Natur, 333, 523

Reines, A. E., Sivakoff, G. R., Johnson, K. E., \& Brogan, C. L. 2011, Natur, 470, 66

Remillard, R. A., \& McClintock, J. E. 2006, ARA\&A, 44, 49

Saxton, R. D., Read, A. M., Esquej, P., et al. 2012, A\&A, 541, A106

Schlegel, D. J., Finkbeiner, D. P., \& Davis, M. 1998, ApJ, 500, 525

Seth, A. C., Cappellari, M., Neumayer, N., et al. 2010, ApJ, 714, 713

Shakura, N. I., \& Sunyaev, R. A. 1973, A\&A, 24, 337

Skinner, G. K., Bedford, D. K., Elsner, R. F., et al. 1982, Natur, 297, 568

Stobbart, A.-M., Roberts, T. P., \& Wilms, J. 2006, MNRAS, 368, 397

Sutton, A. D., Roberts, T. P., \& Middleton, M. J. 2013, MNRAS, 435, 1758

Swartz, D. A., Soria, R., Tennant, A. F., \& Yukita, M. 2011, ApJ, 741, 49

Tao, L., Feng, H., Kaaret, P., Grisé, F., \& Jin, J. 2012, ApJ, 758, 85

Terrier, R., Ponti, G., Bélanger, G., et al. 2010, ApJ, 719, 143

Wang, J., \& Merritt, D. 2004, ApJ, 600, 149

Wilms, J., Allen, A., \& McCray, R. 2000, ApJ, 542, 914 